EPJ Web of Conferences 53, 01006 (2013)

DOI: $10.1051 /$ epjconf/20135301006

(C) Owned by the authors, published by EDP Sciences, 2013

\title{
Mass composition working group report
}

\author{
E. Barcikowski ${ }^{1}$, J. Bellido2,a , J. Belz ${ }^{1}$, Y. Egorov ${ }^{3}$, S. Knurenko ${ }^{3}$, V. de Souza ${ }^{4}$, \\ Y. Tameda ${ }^{5}$, Y. Tsunesada ${ }^{6}$, M. Unger ${ }^{7}$ for the HiRes, Pierre Auger, \\ Telescope Array and Yakutsk Collaborations ${ }^{\mathrm{b}}$
}

${ }^{1}$ Physics Department, University of Utah, USA

2 University of Adelaide, Adelaide, S.A. 5005, Australia

${ }^{3}$ Yu. G. Shafer Institute for Cosmophysical Research and Aeronomy, 31 Lenin Ave., 677980 Yakutsk, Russia

${ }^{4}$ Universidade de São Paulo, Instituto de Física de São Carlos, SP, Brazil

5 Institute for Cosmic Ray Research, the University of Tokyo, Kashiwa, Chiba 277-8582,

Japan

${ }^{6}$ Graduate School of Science and Engineering, Tokyo Institute of Technology, Meguro, Tokyo 152-8550, Japan

${ }^{7}$ Karlsruhe Institute of Technology, Campus North, Institut für Kernphysik, Karlsruhe, Germany

\begin{abstract}
We present a summary of the measurements of mass sensitive parameters at the highest cosmic ray energies done by several experiments. The $X_{\max }$ distribution as a function of energy has been measured with fluorescence telescopes by the HiRes, TA and Auger experiments and with Cherenkov light detectors by Yakutsk. The $\left\langle X_{\max }\right\rangle$ or the average mass $(\langle\ln \mathrm{A}\rangle)$ has been also inferred using ground detectors, such as muon and water Cherenkov detectors. We discuss the different data analyses elaborated by each collaboration in order to extract the relevant information. Special attention is given to the different approaches used in the analysis of the data measured by fluorescence detectors in order to take into account detector biases. We present a careful analysis of the stability and performance of each analysis. The results of the different experiments will be compared and the discrepancies or agreements will be quantified.
\end{abstract}

\section{INTRODUCTION}

In preparation for this meeting, several working groups were formed to establish a common view on the experimental status of measurements at ultra-high energies. Here we report the findings of the mass composition working group consisting of members of the Auger, HiRes, Telescope Array (TA) and Yakutsk collaborations. The aim was to understand and quantify potential differences between different experimental results and to try to discuss the measurements in terms of the cosmic ray mass composition.

A current issue in the field of ultra high energy cosmic rays is that the measurements [1] of the depth of shower maximum from Auger are shallower and less fluctuating than predictions from air shower simulations for a pure proton composition at energies $\gtrsim 10^{19} \mathrm{eV}$. On the other hand, the HiRes and TA results $[3,4]$ in the same energy regions are consistent with QGSJet-II simulations assuming a constant composition dominated by light elements.

\footnotetext{
ae-mail: jbellido@physics.adelaide.edu.au

bFor the full authorlist see Appendix "Collaborations" in this volume.

This is an Open Access article distributed under the terms of the Creative Commons Attribution License 2.0, which permits unrestricted use, distribution, and reproduction in any medium, provided the original work is properly cited.
} 
However, there are important differences in the analysis of the data between Auger, HiRes and TA. In this note we will review the differences between the analyses, compare the different experiment results, and evaluate their compatibility within the experimental statistical and systematic uncertainties.

The main focus of this work is the understanding of the apparent differences between the Auger, HiRes and TA $X_{\max }$ results. Nevertheless, we also discuss in this paper other shower observables which are sensitive to the mass of the primary cosmic rays. The Yakutsk experiment has muon detectors operating and this information is used to infer $\langle\ln A\rangle$ in a complementary way [5]. In a similar way, the Auger collaboration operates an array of water Cherenkov detectors [6] that are able to measure the muonic and electromagnetic signal at the ground level. This signal is used to infer $\langle\ln \mathrm{A}\rangle$.

The challenge for determining the cosmic ray mass composition at the highest energy is our limited knowledge of the hadronic interaction properties. The current hadronic interaction models extrapolate interaction properties measured in particle accelerators at energies more than two orders of magnitude smaller. If we knew the hadronic interaction properties at these higher energies, the interpretation of the data in terms of the primary cosmic ray composition would be straightforward. Despite this limitation, we used the hadronic interaction models to convert the different observables measured by each experiment into $\langle\ln A\rangle$ to allow direct comparison of the measurements.

\section{DIFFERENT APPROACHES TO ANALYSE $X_{\text {MAX }}$ OBSERVATIONS}

The fluorescence detectors (FDs) of the Auger [7], HiRes [8] and TA [9] experiments have a limited field of view (FOV) ranging from about $3^{\circ}$ to $30^{\circ}$ in elevation. This limited FOV introduces a detector bias depending on the shower geometry: For close by showers, the lack of a high elevation FOV prevents the observation of shallow showers and, therefore, deeper showers are favored in the event selection. Moreover, deep near-vertical showers may reach their maximum below the FOV range (or even below ground level) and therefore the ensemble of selected showers is artificially enriched with shallow showers. To address this problem the collaborations follow two different approaches.

\subsection{The auger approach}

The goal of the Auger Collaboration is to publish $\left\langle X_{\max }\right\rangle$ and $\operatorname{RMS}\left(X_{\max }\right)$ values with minimal detector bias, that are close to the moments of the undistorted distribution. This procedure is only limited by systematic uncertainties. The advantage of this proposal is that the published results can be compared directly with model expectations, without the need of any knowledge of the detector and, therefore, also to simulations with hadronic interaction models and or composition hypotheses that were not available at the time of publication.

In order to measure an unbiased $X_{\max }$ distribution, it is necessary to select events based only on their arriving geometry and energy (i.e. not using any information of their $X_{\max }$ value). The basic idea is to select showers with geometries that will allow $X_{\max }$ to be inside a detector FOV that is "wide enough" to cover the true $X_{\max }$ distribution. In order to choose the appropriate geometries to be considered in the $X_{\max }$ analysis, it is necessary to have a rough idea of what the range of the $X_{\max }$ distribution is. The idea is to use the data themselves to determine this range for each energy bin.

Given the geometry and energy of an event, the range in $X_{\max }$ for which a shower can be detected with good quality can be predicted with a semi-analytical calculation that takes into account the atmospheric attenuation and the optical efficiency of the detector. The result of such a calculation is an $X_{\max }$-range, $X_{\mathrm{fov}}^{\min }$ to $\mathrm{X}_{\mathrm{fov}}^{\max }$, for which a shower will be accepted for the data analysis defining the effective (as opposed to geometrical) FOV of the telescopes.

Fig. 1 shows the scatter plots of the measured $X_{\max }$ between $10^{18.0}$ and $10^{18.2} \mathrm{eV}$ as a function of the estimated $X_{\text {fov }}^{\min }$ and $X_{\text {fov }}^{\max }$ as well as the dependence of the average $X_{\max }$ values on these variables. The two plots clearly illustrate the resulting bias that is introduced when $X_{\text {fov }}^{\min }$ is too deep (panel on the left) or when $\mathrm{X}_{\mathrm{fov}}^{\max }$ is too shallow (panel on the right). The Auger Collaboration analysis procedure uses 


\section{UHECR 2012}
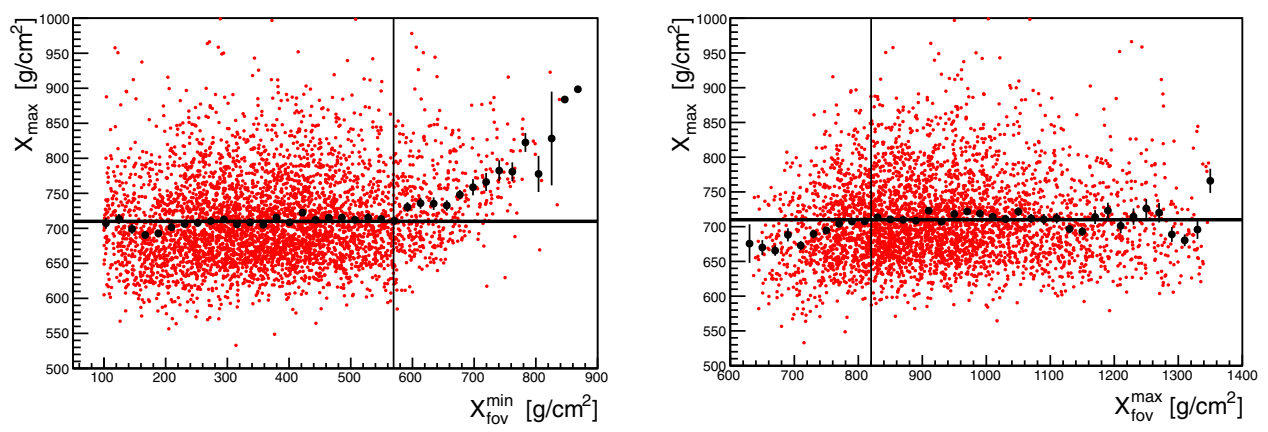

Figure 1. Scatter plots of the measured $X_{\max }$ between $10^{18.0}$ and $10^{18.2} \mathrm{eV}$ as a function of the event-by-event field of view boundaries $X_{\mathrm{fov}}^{\min }$ and $\mathrm{X}_{\mathrm{fov}}^{\max }$. The mean $X_{\max }$ values as a function of $\mathrm{X}_{\mathrm{fov}}^{\min }$ and $\mathrm{X}_{\mathrm{fov}}^{\max }$ are superimposed as large black dots. The horizontal lines denote the asymptotic $\left\langle X_{\max }\right\rangle$ value far away from the field of view boundary. The range where the $\left\langle X_{\max }\right\rangle$ values are consistent with this line defines the unbiased region. The vertical lines indicate the limits of fiducial field of view that result in an unbiased $X_{\max }$ measurement.

Table 1. Number of events above $10^{18.2} \mathrm{eV}$ and $10^{19.0} \mathrm{eV}$ (Fig. 6). In this table we have included the number of reconstructed Auger events that survived all the quality cuts (i.e. number of events prior to the application of the field-of-view cuts). The energy distribution of these data is not shown in Fig. 6. The total number of events that the HiRes collaboration has used for the $X_{\max }$ analysis above $10^{18.2} \mathrm{eV}$ is 815 . However, after the application of the energy normalization (normalized to the TA energy scale) across experiments, 798 HiRes events remained with energies above $10^{18.2} \mathrm{eV}$ (17 events ended up with energies below this). The HiRes collaboration applies a cut on $\mathrm{Rp}$ to reduce the detector bias effect. This table shows the number of events before applying this Rp cut.

\begin{tabular}{|l|c|c|c|c|c|c|}
\hline & $\begin{array}{c}\text { Auger } \\
\text { standard cuts }\end{array}$ & $\begin{array}{c}\text { Auger } \\
\text { without FOV cuts }\end{array}$ & $\begin{array}{c}\text { HiRes } \\
\text { standard cuts }\end{array}$ & $\begin{array}{c}\text { HiRes } \\
\text { no Rp cuts }\end{array}$ & TA & Yakutsk \\
\hline $\mathrm{E}>10^{18.2} \mathrm{eV}$ & 5138 & 11343 & 798 & 1306 & 279 & 412 \\
\hline $\mathrm{E}>10^{19.0} \mathrm{eV}$ & 452 & 709 & 123 & 143 & 67 & 22 \\
\hline
\end{tabular}

these graphs for each energy bin in order to determine what ranges of $X_{\text {fov }}^{\min }$ and $X_{\text {fov }}^{\max }$ values allow for an unbiased sampling of the $X_{\max }$ distributions as a function of energy $[10,11]$. The vertical lines in Figure 1 indicates the appropriate limits for $X_{\text {fov }}^{\min }$ and $X_{\text {fov }}^{\max }$. These limits determine the fiducial field-ofview cuts. These fiducial field-of-view cuts are optimized independently for the data and for each Monte Carlo (MC) composition. Different MC compositions (i.e. different $X_{\max }$ distributions) have been used to test this algorithm $[10,11]$ and the reconstructed $\left\langle X_{\max }\right\rangle$ values were found consistent with the MC input within statistical uncertainties.

The Auger collaboration applies identical quality cuts to data and Monte Carlo, which is similar to the approach shared by HiRes and TA (in the analysis described below) in which equivalent cuts are applied to data and Monte Carlo throughout. The additional field-of-view cuts - for which there is no direct analog in the HiRes or TA analysis - are optimized independently for the data and for each MC composition, using the same algorithm for this optimization. The motivation behind this choice is to allow optimization of the field-of-view cuts without making any a priori assumptions as to the range of the $X_{\max }$ distribution.

Above $10^{18.2} \mathrm{eV}$, the application of the field-of-view cuts reduce the Auger statistics by half (Table 1). The severity of these field-of-view cuts (for reducing the statistics) puts a constraint on the minimum number of events required for this approach.

\subsection{The HiRes and TA approach}

The HiRes and TA collaborations do not apply field-of-view cuts. This means that they do not attempt to measure the unbiased $\left\langle X_{\max }\right\rangle$ in the atmosphere, instead they quote the $\left\langle X_{\max }\right\rangle$ as measured in the 
detector (we will refer it as $\left\langle X_{\max }^{\operatorname{meas}}\right\rangle$ ). These two $\left\langle X_{\max }\right\rangle$ values can differ significantly depending on the intrinsic $X_{\max }$ distribution, or depending on the zenith angle distribution of the events (as shown in Figs. 8(b) and 8(c)). However, this should not affect the HiRes/TA $X_{\max }$ composition analysis, because an accurate detector modeling is used for predicting the $\left\langle X_{\max }^{\operatorname{mea}}\right\rangle$ observations for a given composition.

In order to understand the acceptance and reconstruction biases arising from the inherent field-ofview limitations of a fluorescence detector, both the HiRes and TA collaborations focus on accurate detector modeling through the use of a detailed detector Monte Carlo. Air showers are generated using CORSIKA [12] and several hadronic interaction models including QGSJet01 [13], QGSJetII [14] and SIBYLL [15, 16]. Shower libraries are created in which the number of particles as a function of slant depth is recorded for a large number of air showers induced by different primary masses.

In the detector simulation, an event is drawn from the library and assigned a random core location, zenith and azimuthal angle. The fluorescence light is propagated from the shower to the detector, with attenuation simulated via the use of an empirically determined atmospheric database. Ray tracing is performed to determine the photoelectron response of individual PMT's in the fluorescence camera. The trigger algorithms are simulated, and if the trigger conditions are satisfied the Monte Carlo event is written to disk in the identical format as real data, allowing study by the same analysis chain.

A number of control distributions are checked for agreement between data and Monte Carlo in order to assure that all detector effects are accurately described by the simulation. Particular attention is paid to those distributions - e.g. first and last viewed depth of the shower - which touch closely on the detector biasing issue. Finally, to extract information about composition the observed $X_{\max }$ distributions in the data are compared to the "observed" $X_{\max }$ distributions for Monte Carlo events which pass identical event selection criteria.

In summary, in order to estimate an average mass composition, the $\left\langle X_{\max }\right\rangle$ measured by Auger can be compared directly with the predictions from air shower simulations (within remaining systematic uncertainties). In the case of HiRes and TA, the measured $\left\langle X_{\max }^{\text {meas }}\right\rangle$ should be compared with the $\left\langle X_{\max }^{\text {meas }}\right\rangle$ obtained from a convolution of simulated showers with a model of the detector, atmosphere and reconstruction.

It is important to note that the $\left\langle X_{\max }\right\rangle$ measurements by Auger and Yakutsk cannot be compared directly with the $\left\langle X_{\max }^{\text {meas }}\right\rangle$ published by HiRes and TA. In Sec. 7 we will transform these $\left\langle X_{\max }\right\rangle$ and $\left\langle X_{\max }^{\text {meas }}\right\rangle$ to $\langle\ln \mathrm{A}\rangle$ to compare the different experiment results.

\section{YAKUTSK MEASUREMENT OF THE $X_{\text {MAX }}$ DISTRIBUTIONS}

The determination of $X_{\max }$ in individual showers is based on the measurement of the Cherenkov light flux at different core distances $\mathrm{Q}(\mathrm{r})$ :

1. by the parameter $\mathrm{p}=\lg (\mathrm{Q}(200) / \mathrm{Q}(500))$;

2. reconstruction of a shower development curve from the lateral distribution of Cherenkov light [17];

3. measurement of the Cherenkov light pulse width at a fixed core distance;

4. by recording a Cherenkov track with a differential detector based on camera obscura.

The sensitivity of these techniques is described in [18]. The accuracy of $X_{\max }$ determination in individual showers was estimated in a simulation of EAS characteristics measurements at the array involving MC methods and amounted to $30-45 \mathrm{~g} / \mathrm{cm}^{2}, 35-55 \mathrm{~g} / \mathrm{cm}^{2}, 15-25 \mathrm{~g} / \mathrm{cm}^{2}$, $35-55 \mathrm{~g} / \mathrm{cm}^{2}$ respectively for the first, second, third and fourth methods. The total error of $X_{\max }$ estimation included errors associated with core location, atmospheric transparency during the observational period, hardware fluctuations and mathematical methods used to calculate a main parameters. 


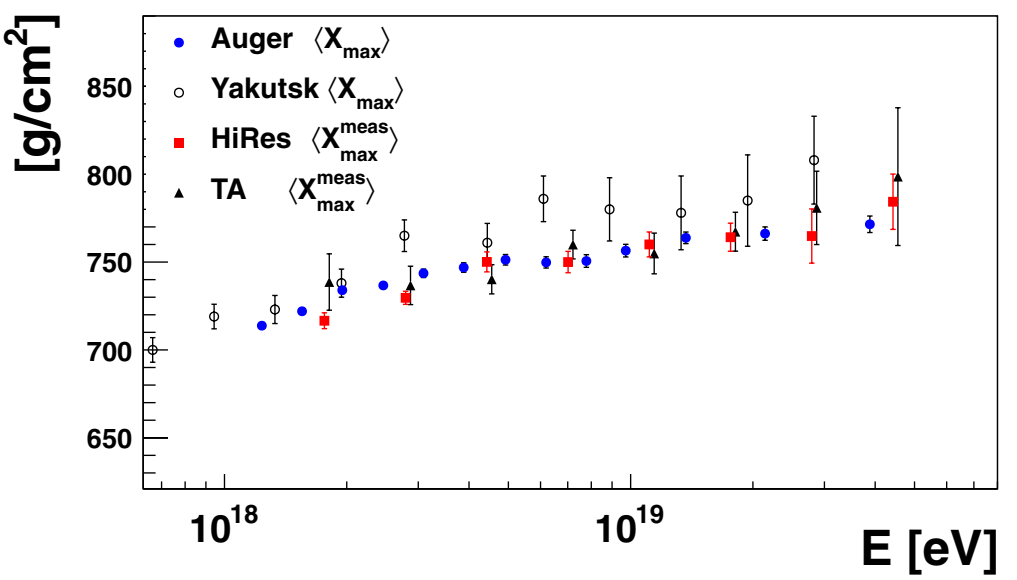

Figure 2. $\left\langle X_{\max }\right\rangle$ measured by Auger and Yakutsk, together with the $\left\langle X_{\max }^{\text {meas }}\right\rangle$ as measured by HiRes and TA. Data points are shifted to a common energy scale (text for details).
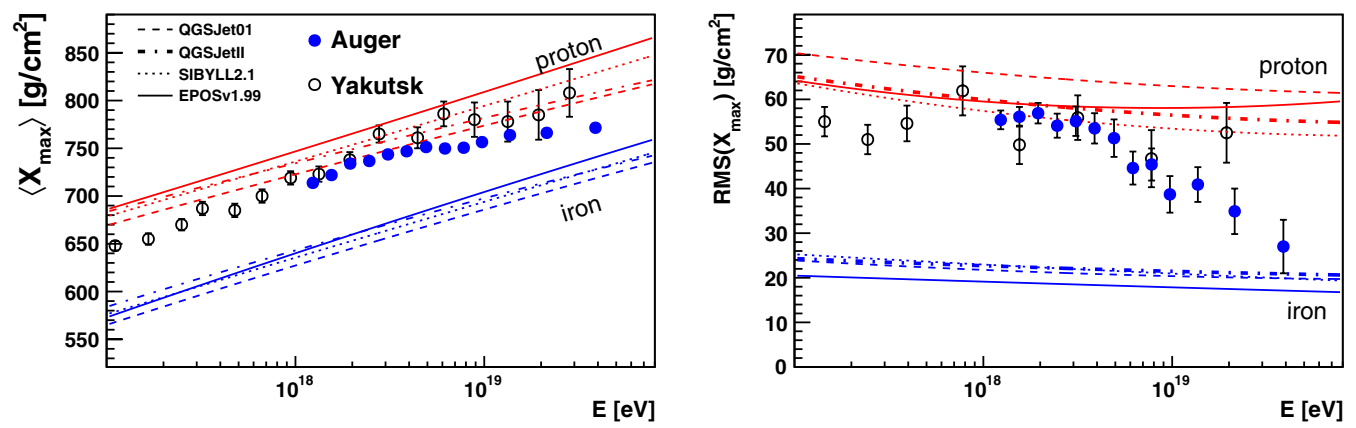

Figure 3. Measured $\left\langle X_{\max }\right\rangle$ (left) and $\operatorname{RMS}\left(X_{\max }\right)$ (right) for the Auger and Yakutsk experiments. The lines indicate the $\left\langle X_{\max }\right\rangle$ expectations for proton and iron compositions using different hadronic interaction models. Notice that the highest energy bin for Yakutsk contains only 3 events (Fig. 6).

\section{COMPARING DIFFERENT $X_{\mathrm{MAX}}$ MEASUREMENTS}

Figure 2 shows the $\left\langle X_{\max }\right\rangle$ measured by Auger [19] and Yakutsk [20], together with the $\left\langle X_{\max }^{\text {meas }}\right\rangle$ as measured by HiRes [3] and TA [4]. The observed agreement between the measured $\left\langle X_{\max }\right\rangle$ and $\left\langle X_{\max }^{\text {max }}\right\rangle$ is not expected.

At this meeting, the energy spectrum working group has compared the shape of the energy spectrum from the Auger, Yakutsk, HiRes and TA experiments and has produced a table with normalization factors [22]. For the plots presented here, we have normalized the energy scales to an energy scale that is half way between the Auger and TA energy scales. The normalization factors that we have used are 1.102 for Auger, 0.55 for Yakutsk, 0.883 for HiRes and 0.908 for TA. Later in Sec. 7 we will evaluate the compatibility of the different results. We will transform $\left\langle X_{\max }\right\rangle$ and $\left\langle X_{\max }^{\text {meas }}\right\rangle$ to $\langle\ln \mathrm{A}\rangle$ for meaningful comparisons.

Figure 3 shows the measured $\left\langle X_{\max }\right\rangle$ (panel on the left) and $\operatorname{RMS}\left(X_{\max }\right)$ (panel on the right) for the Auger and Yakutsk experiments. Since both experiments published $\left\langle X_{\max }\right\rangle$ values with minimum detector bias, we can compare both of them with the model expectations. The same holds true for the measurements of the shower-to-shower fluctuations, where both experiments corrected the measurements for the detector resolution. The lines indicate the predictions from air shower simulations 

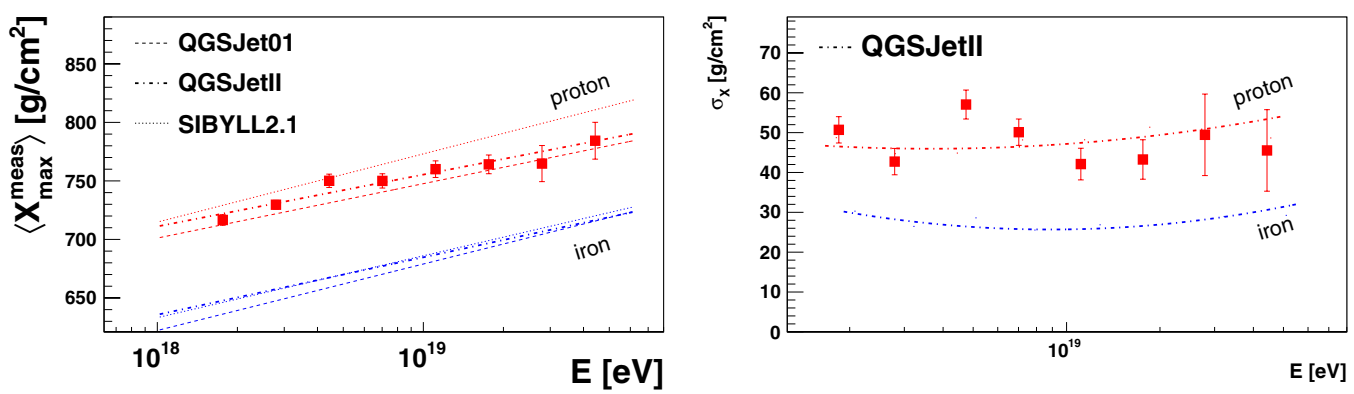

Figure 4. The $\left\langle X_{\max }^{\text {meas }}\right\rangle$ (left) and $\operatorname{RMS}\left(X_{\max }\right)$ (right) as measured by the HiRes experiment. The lines are the corresponding $\left\langle X_{\max }^{\max }\right\rangle$ and $\sigma_{X}$ expectations for proton and iron compositions. The different line types correspond to different models.

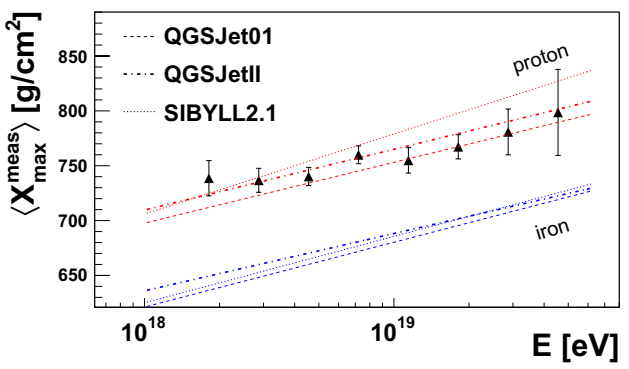

Figure 5. The $\left\langle X_{\max }^{\text {meas }}\right\rangle$ measured by the TA experiment. The lines are the corresponding $\left\langle X_{\max }^{\text {meas }}\right\rangle$ expectations for proton and iron primaries. The different line types correspond to different models.

for proton and iron compositions. There are different line types corresponding to different high energy hadronic interaction models: QGSJet-01, QGSJet-II, SIBYLL2.1 and EPOSv1.99.

The HiRes collaboration chooses a fluctuation estimator that differs from the one published by Auger and Yakutsk. Whereas the latter use simply the standard deviation (denoted by RMS $\left(X_{\max }\right)$ ), HiRes uses the width of an unbinned likelihood fit with a Gaussian to the distribution truncated at $2 \times$ RMS, denoted by $\sigma_{X}$.

Figure 4 shows the $\left\langle X_{\max }^{\text {meas }}\right\rangle$ and $\sigma_{X}$ as measured by HiRes. The lines are the corresponding $\left\langle X_{\max }^{\text {meas }}\right\rangle$ and $\sigma_{X}$ expectations for proton and iron compositions. The different line types correspond to different models (QGSJet-01, QGSJet-II, SIBYLL2.1).

Figure 5 shows the corresponding $\left\langle X_{\max }^{\max }\right\rangle$ observation and expectation for the TA experiment. Currently the TA experiment does not have enough statistics to quantify the width of the $X_{\max }$ distributions at the highest energies.

Figure 6 shows the energy distributions and total number of events that survived the selection cuts at each experiment. For this Figure, the energy scales have been normalized to the TA energy scale. A summary of Figure 6 is shown in Table 1.

The $\left\langle X_{\max }\right\rangle$ measurements from Yakutsk (Fig. 3), HiRes (Fig. 4) and TA (Fig. 5) experiments are consistent with the QGSJet predictions for a constant proton composition at all energies above $10^{18} \mathrm{eV}$, whereas the $\left\langle X_{\max }\right\rangle$ measurements from the Pierre Auger Observatory are significantly shallower than these predictions above a few EeV (cf. left panel Fig. 3).

At the same time, the width of the $X_{\max }$ distribution measured by Auger gets narrower above a few $\mathrm{EeV}$ and the Yakutsk measurements of the fluctuations are consistent with the Auger up to about $10^{19} \mathrm{eV}$. Yakutsk has one measurement of the $X_{\max }$ width above $10^{19} \mathrm{eV}$ and it is wider than the Auger one by 


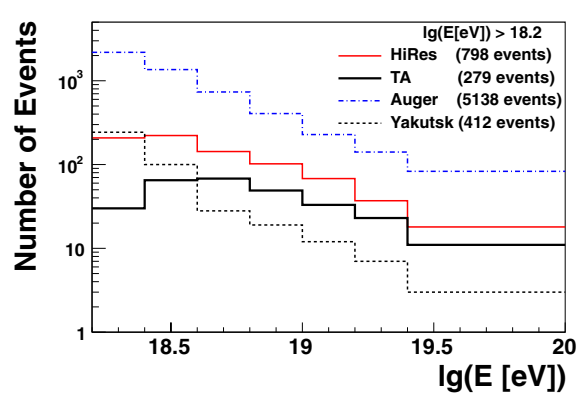

Figure 6. Number of events that survived the selection cuts as a function of energy. For this plot the energies have been normalized to the TA energy scale.
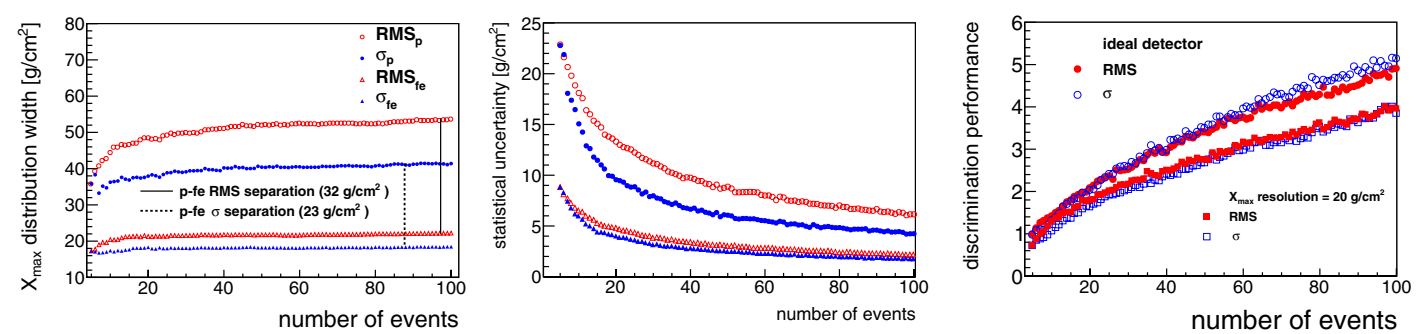

Figure 7. Left: Average value of the measured RMS and $\sigma_{X}$ as a function of the number of events in the sample. (middle) Statistical uncertainty of the measured RMS and $\sigma_{X}$. Right: Sensitivity or discrimination power for the RMS and $\sigma_{X}$ approaches.

about 3 standard deviations (right panel Fig. 3). The $X_{\max }$ distribution widths measured by HiRes at energies above $2 \times 10^{19} \mathrm{eV}$, while consistent with pure proton composition have large statistical errors and do not definitely exclude a heavier composition (right panel Fig. 4).

\subsection{A toy MC to evaluate the performance of different fluctuation measures}

The $X_{\max }$ distribution is expected to have an asymmetric long tail due to the exponential nature of the depth of the first interaction. Both, $\operatorname{RMS}\left(X_{\max }\right)$ and $\sigma_{X}$, could be under-estimated if they are sampled with a small number of events. We have used a toy MC simulation to evaluate the performance of both methods for the quantification of the shower-to-shower fluctuations of $X_{\max }$.

We have generated random samples of "N" events with $X_{\max }$ distributed following the expectation for $10^{19} \mathrm{eV}$ proton and iron showers using the QGSJet-II model. Fig. 7 (left panel) shows the measured RMS and $\sigma_{X}$ as a function of the number of events "N". As can be seen, both approaches show a bias in the measured $X_{\max }$ distribution width when the number of events in the sample is small. For samples with at least 30 events, this bias is negligible. Even for smaller statistics, the magnitude of the bias from under-sampling is negligible compared with the statistical uncertainty. The statistical uncertainties for the measured RMS and $\sigma_{X}$ are shown in the middle panel of Fig. 7.

When using the RMS to quantify the $X_{\max }$ distribution width, the separation between proton and iron compositions is larger. On the other hand, the associated statistical uncertainties are smaller when using $\sigma_{X}$. In order to evaluate the performance of using the RMS or the $\sigma_{X}$ to quantify the width of the $X_{\max }$ distribution, we have computed a performance indicator defined as:

$$
\text { sensitivity }=\frac{\Delta \text { width }(\mathrm{p} / \mathrm{Fe})}{\sqrt{\sigma_{\mathrm{p}}^{2}+\sigma_{\mathrm{Fe}}^{2}}},
$$




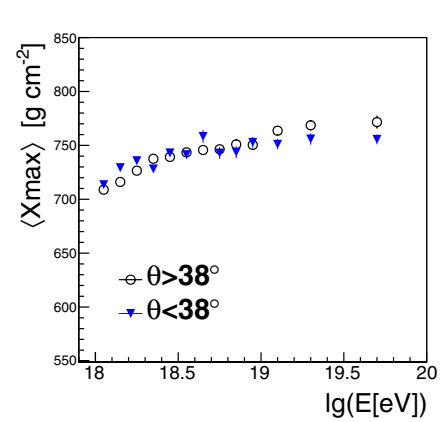

(a) Auger $\left\langle X_{\max }\right\rangle$

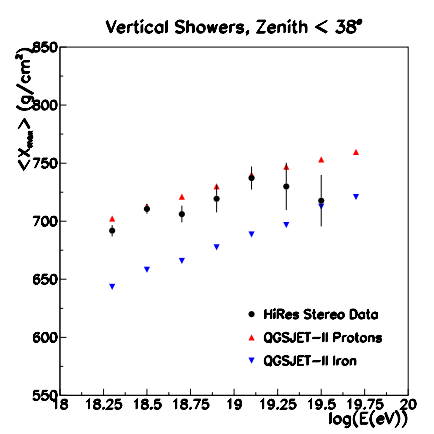

(b) HiRes $\left\langle X_{\max }^{\operatorname{meas}}\right\rangle$

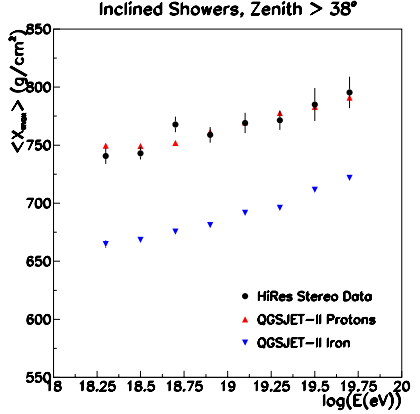

(c) HiRes $\left\langle X_{\max }^{\max }\right\rangle$

Figure 8. The $\left\langle X_{\max }\right\rangle$ and $\left\langle X_{\max }^{\text {meas }}\right\rangle$ for Auger and HiRes using showers from different zenith angle ranges.

where $\Delta$ width $(\mathrm{p} / \mathrm{Fe})$ denotes the average difference between the $X_{\max }$ distribution width for proton and iron (measured using the $\operatorname{RMS}\left(X_{\max }\right)$ or $\sigma_{X}$ respectively), and $\sigma_{p}$ and $\sigma_{f e}$ are the corresponding statistical uncertainties of the fluctuation measurements.

Figure 7 shows the computed sensitivities (right panel). It turns out that the sensitivity of both approaches is basically equivalent at all ranges of number of events. We have also introduced a $20 \mathrm{~g} / \mathrm{cm}^{2}$ $X_{\max }$ resolution effect to compute the sensitivity. As expected, this reduces the sensitivity in both cases, but does not change the equivalence of both approaches.

\section{STABILITY OF THE $X_{\text {MAX }}$ OBSERVATIONS AND CROSS CHECKS}

In this section we want to show how stable the $X_{\max }$ distribution measurements are.

We have checked whether the measured $X_{\max }$ distributions depend on the the zenith angle. Vertical showers are more affected by the ground level truncation of the distribution and, moreover, the fluorescence light has to traverse denser regions of the atmosphere to the detector.

Due to the analysis strategy used in Auger, there is no significant difference between the vertical and inclined $\left\langle X_{\max }\right\rangle$ measurements, as is illustrated in Fig. 8(a)).

In the case of HiRes, there is about $40 \mathrm{~g} / \mathrm{cm}^{2}$ difference between the $\left\langle X_{\max }\right\rangle$ measured in two zenith angle intervals, as can be seen by comparing Figures 8(b) and 8(c)). This difference is however well reproduced by the detector simulation and for both zenith angle intervals the data are compatible with the proton prediction from QGSJet-II.

The Auger collaboration has used MC data to evaluate the flatness of the detector acceptance as a function of the depth of $X_{\max }$. Reference [24] shows that this acceptance becomes flat after the application of the field-of-view cuts.

Another way to cross check that there is a homogeneous acceptance in the Auger $\left\langle X_{\max }\right\rangle$ analysis, which is independent of the shower composition, is by comparing data and MC energy distributions resulting after the fiducial cuts. Figure 9 shows the energy distribution of the Auger data from [1] compared with the distributions for proton and iron. For Fig. 9(a), the MC distributions were obtained applying quality cuts only (i.e. without applying fiducial cuts). The MC events were re-weighted at generator level to match the spectral shape of the CR flux measured by the Auger surface detector [23] and normalized to the data at $10^{18.6} \mathrm{eV}$. As expected, the spectral shape of the MC without fiducial selection does not match the data. It is because the acceptance depends on the composition (or, more precisely, on the distribution of shower maxima in the atmosphere). After application of the fiducial 


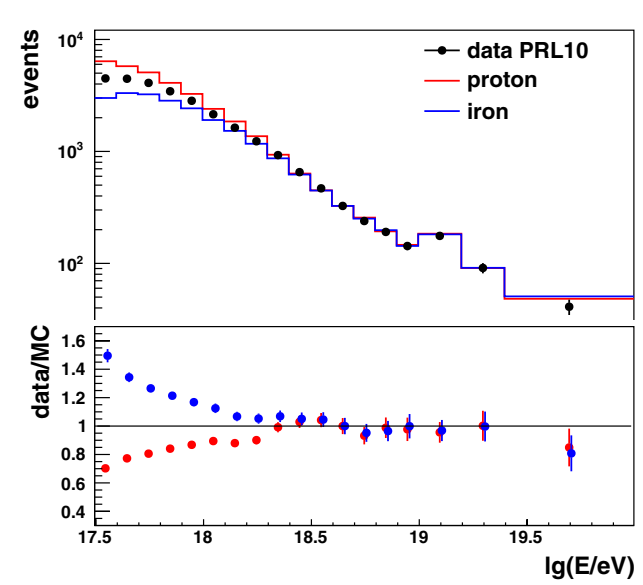

(a) without fiducial cuts (quality cuts only)

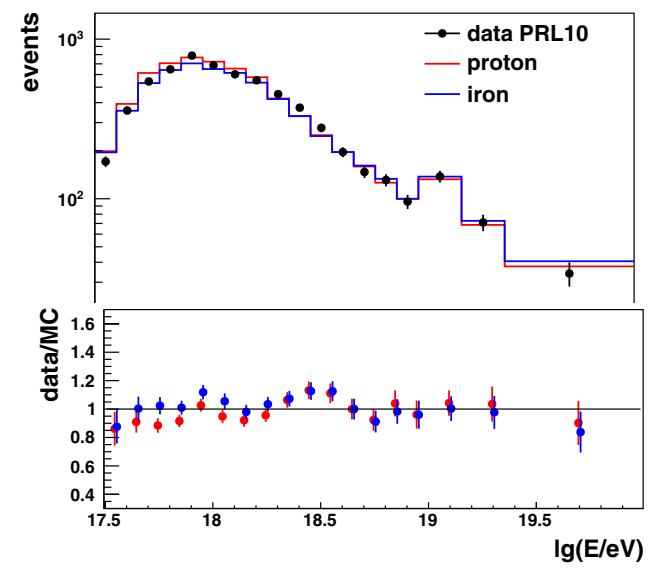

(b) with fiducial cuts

Figure 9. Energy distribution of selected events before and after applying the fiducial cuts. MC histograms have been normalized to the data at $10^{18.6} \mathrm{eV}$.

field-of-view cuts, the spectral shapes of both, the proton and iron simulations, agree well with the data (Fig. 9(b)).

To check if there could be a difference in interpreting the data due to different analysis strategies, currently the TA and Auger collaborations are separately working on their data interpretation using both analysis strategies.

\section{VALIDITY OF THE DETECTOR MONTE CARLO}

The $X_{\max }$ analysis approaches followed by Auger, TA and HiRes require some information from detector Monte Carlo simulations.

\subsection{Auger}

For the Auger approach, the detector MC simulations are used to estimate the average $X_{\max }$ reconstruction bias and the average $X_{\max }$ resolution as a function of energy. They are used to correct the observed $\left\langle X_{\max }\right\rangle$ and $\operatorname{RMS}\left(X_{\max }\right)$ values respectively. After applying fiducial volume cuts, the correction on $\left\langle X_{\max }\right\rangle$ is smaller than $4 \mathrm{~g} / \mathrm{cm}^{2}$, and the average $X_{\max }$ resolution is about $20-25 \mathrm{~g} / \mathrm{cm}^{2}$.

The Auger collaboration has used stereo events to cross check the validity of its detector simulations. Stereo events have been simulated, reconstructed and selected in the same way as data. The advantage of using stereo events is that showers are reconstructed almost independently using each of the FD observations (they are not completely independent because they use the same surface station for the hybrid reconstruction of the geometry). As a result we obtain two measurements of the shower parameters. From the comparison of these two sets of shower parameters the corresponding resolutions are estimated. The resolution in $X_{\max }$ depends on the characteristics of the showers (such as geometry and energy). So, the resolution obtained using stereo events is not a representative $X_{\max }$ resolution of regular hybrid events (that are on average of lower energy than stereo events). However, the $X_{\max }$ resolution obtained with data and MC stereo events has to be consistent if the detector simulation is working correctly. Figure 10(a) shows the consistency of the $X_{\max }$ resolution obtained using data and simulated stereo events. 


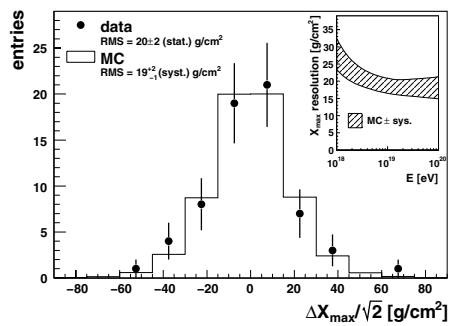

(a) Auger

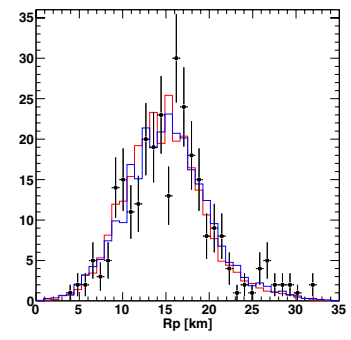

(b) TA

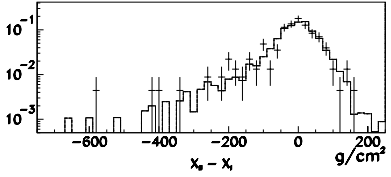

(c) HiRes

Figure 10. Validating the detector MC simulations. (a) Auger $X_{\max }$ difference of stereo events for data and MC. The $\left\langle X_{\max }\right\rangle$ resolution is displayed as a function of energy in the inset. (b) TA $R_{p}$ distribution for data (black points) and MC (solid lines). The red and blue lines are for QGSJet-II proton and iron composition. (c) Difference between HiRes-II $\left(X_{I I}\right)$ and HiRes-I $\left(X_{I}\right) X_{\max }$ for HiRes stereo data (points) overlaid with QGSJet-II proton Monte Carlo calculations.

\subsection{HiRes and TA}

For the TA and HiRes approach, the detector MC simulations are used to estimate the expected $X_{\max }$ distributions after considering the detector effects. These expectations are estimated for different cosmic ray primaries. Then, the expected and observed $X_{\max }$ distributions are compared to infer the average cosmic ray composition.

Figure 10(b) shows the $R_{p}$ distribution for TA data and for MC calculations. Figure 10(c) shows the $X_{\max }$ difference between HiRes-II $\left(X_{I I}\right)$ and HiRes-I $\left(X_{I}\right)$ for HiRes stereo data (points) overlaid with QGSJet-II proton Monte Carlo calculations. The asymmetry is caused by HiRes-I covering only half the range in elevation angle. The Gaussian width of the peak is $44 \mathrm{~g} / \mathrm{cm}^{2}$, setting an upper limit of $31 \mathrm{~g} / \mathrm{cm}^{2}$ for the HiRes-II $X_{\max }$ resolution. Monte Carlo studies indicate that the actual HiRes-II $X_{\max }$ resolution is better than $25 \mathrm{~g} / \mathrm{cm}^{2}$ over most of the HiRes energy range.

\section{COMPARISON OF $X_{\text {MAX }}$ RESULTS FROM DIFFERENT EXPERIMENTS}

In order to make sensible comparisons between experiments, we have used the observed $\left\langle X_{\max }\right\rangle$ values to infer the average logarithmic mass, $\langle\ln \mathrm{A}\rangle$, using.

$$
\langle\ln A\rangle=\frac{\left\langle X_{\max }\right\rangle_{\mathrm{p}}-\left\langle X_{\max }\right\rangle_{\mathrm{data}}}{\left\langle X_{\max }\right\rangle_{\mathrm{p}}-\left\langle X_{\max }\right\rangle_{\mathrm{Fe}}} \ln 56,
$$

Similarly, one can transform $\left\langle X_{\max }^{\text {mas }}\right\rangle$ into $\langle\ln \mathrm{A}\rangle$ by replacing $\left\langle X_{\max }\right\rangle$ with $\left\langle X_{\max }^{\text {meas }}\right\rangle$ in this equation although this is only correct as a first order approximation. This is because $\left\langle X_{\max }^{\text {max }}\right\rangle$ does not correlate linearly with $\langle\ln \mathrm{A}\rangle$ as $\left\langle X_{\max }\right\rangle$ does [2].

When we transformed the measured $\left\langle X_{\max }\right\rangle$ into $\langle\ln \mathrm{A}\rangle$, we used the expected $\left\langle X_{\max }\right\rangle$ values for proton and iron obtained directly from Conex simulations. On the other hand, when we transformed the measured $\left\langle X_{\max }^{\text {meas }}\right\rangle$ values, we used the expected $\left\langle X_{\max }^{\text {meas }}\right\rangle$ values for proton and iron extracted from the simulation including the detector.

Figure 11 shows the $\langle\ln \mathrm{A}\rangle$ estimated using the QGSJet-II and SIBYLL interaction models. The shaded regions indicate the range of the corresponding systematic uncertainties that were propagated from the systematic uncertainties in $\left\langle X_{\max }\right\rangle\left(12 \mathrm{~g} / \mathrm{cm}^{2}\right.$ for Auger and TA, $20 \mathrm{~g} / \mathrm{cm}^{2}$ for Yakutsk, and $6 \mathrm{~g} / \mathrm{cm}^{2}$ for HiRes).

HiRes quotes systematics broken down into a $3.4 \mathrm{~g} / \mathrm{cm}^{2}$ shift in the mean and an uncertainty of $3.2 \mathrm{~g} / \mathrm{cm}^{2}$ decade in the elongation rate. For the purposes of the present comparison, we have combined 


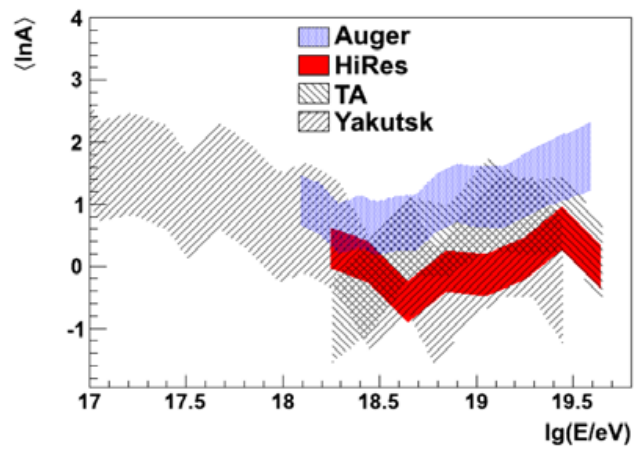

(a) using QGSJet-II model.

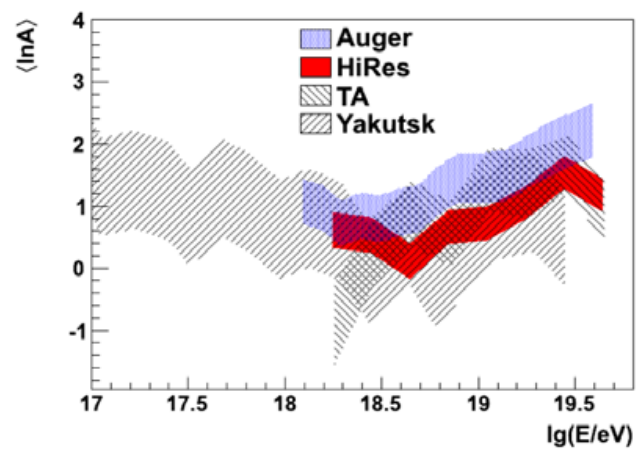

(b) using SIBYLL model.

Figure 11. Comparing the average composition $(\langle\ln \mathrm{A}\rangle)$ estimated using Auger, HiRes, TA and Yakutsk data. The shaded regions correspond to the systematic uncertainty ranges. To infer the average composition from $\left\langle X_{\max }\right\rangle$, QGSJet-II and SIBYLL models have been used.

the two HiRes uncertainties into a single number by adding in quadrature the uncertainty in the mean and the shift due to a $1 \sigma$ variation in slope over 1.6 decades of energy.

All the systematic uncertainties (on the measured $\left\langle X_{\max }\right\rangle$ ) used in this work correspond to each experiment's quoted value. This working group has not attempted to validate those values.

At ultra-high energies, the Auger data suggest a larger $\langle\ln \mathrm{A}\rangle$ than all other experiments. The Auger results are consistent within systematic uncertainties with TA and Yakutsk, but not fully consistent with HiRes. HiRes is compatible with the Auger data only at energies below $10^{18.5} \mathrm{eV}$ when using QGSJetII (Fig. 11(a)), and when using SIBYLL model, Auger and HiRes become compatible within a larger energy range (Fig. 11(b)).

Comparing Figs. 11(a) and 11(b) we find that the level of incompatibility between Auger and HiRes data depends on the model used to interpret the $\left\langle X_{\max }\right\rangle$ observations. Different models predict different ranges of $X_{\max }$ values for proton and iron cosmic rays, and depending on how these predictions compare with the range of $X_{\max }$ values that could be inside the FOV of the detector, the $\left\langle X_{\max }^{\operatorname{meas}}\right\rangle$ (observed by HiRes) could be more or less different to the intrinsic $\left\langle X_{\max }\right\rangle$, changing the interpretation of $\left\langle X_{\max }^{\text {meas }}\right\rangle$. The HiRes results are compatible in every way with the interpretation that the composition is light, i.e. lighter than the CNO group of elements. The Auger $\left\langle X_{\max }\right\rangle$ and $\operatorname{RMS}\left(X_{\max }\right)$ results do not allow this interpretation.

Figure 2 shows that the $\left\langle X_{\max }\right\rangle$ observed by Auger and the $\left\langle X_{\max }^{\operatorname{mea}}\right\rangle$ observed by HiRes and TA are similar. Is there any physical reason that the $\left\langle X_{\max }\right\rangle$ for Auger and the $\left\langle X_{\max }^{\operatorname{meas}}\right\rangle$ for HiRes and TA are all similar, or is it just coincidence? A direct way of checking the Auger and HiRes/TA compatibility would be to simulate a hypothetical composition which had the same $X_{\max }$ distributions as observed by Auger. Then this composition would be propagated through the HiRes and TA detector simulations and the expected $\left\langle X_{\max }^{\text {meas }}\right\rangle$ computed. So, we could compare directly the expected and observed $\left\langle X_{\max }^{\text {meas }}\right\rangle$ to evaluate the compatibility of the Auger and HiRes/TA observations (this is work in progress).

We have also evaluated how the average logarithmic mass estimated by the experiments evolves as a function of energy. Currently there are two different models suggested by the Auger and HiRes collaborations. The $\left\langle X_{\max }\right\rangle$ and $\operatorname{RMS}\left(X_{\max }\right)$ observed by the Auger experiment suggest that the composition might be becoming lighter with energy up to $10^{18.3} \mathrm{eV}$, and heavier above this energy. On the contrary, the $\left\langle X_{\max }\right\rangle$ and $\operatorname{RMS}\left(X_{\max }\right)$ observed by the HiRes experiment is consistent with a constant composition (light composition) all along the observed energy range. We have evaluated both, the Auger and HiRes composition models using Auger, HiRes, TA and Yakutsk data (only statistical uncertainties were considered for this evaluation). The results are summarized in Tables 2 and 3. 
Table 2. Fitting a horizontal line to the $\langle\ln A\rangle$ as a function of energy.

\begin{tabular}{|c|c|c|c|c|}
\hline & Auger & HiRes & TA & Yakutsk \\
\hline Constant $\langle\ln \mathrm{A}\rangle$ & $1.11 \pm 0.03$ & $0.6 \pm 0.1$ & $0.8 \pm 0.2$ & $0.3 \pm 0.2$ \\
\hline$\chi^{2} / n d f$ & $133.6 / 10$ & $4.4 / 7$ & $9.8 / 7$ & $7.7 / 7$ \\
\hline
\end{tabular}

Table 3. Fitting a broken line to the $\langle\ln \mathrm{A}\rangle$ as a function of energy. For HiRes, TA and Yakutsk the slopes were fixed to the Auger ones, only the position of the breaking points were fitted.

\begin{tabular}{|c|c|c|c|c|}
\hline & Auger & HiRes & TA & Yakutsk \\
\hline First Slope & $-1.0 \pm 0.3$ & -1.0 & -1.0 & -1.0 \\
\hline Second Slope & $1.3 \pm 0.1$ & 1.3 & 1.3 & 1.3 \\
\hline $\lg \left(E_{\text {break }} / \mathrm{eV}\right)$ & $18.43 \pm 0.04$ & $18.65 \pm 0.07$ & $18.26+0.14 /-\infty$ & $18.62 \pm 0.14$ \\
\hline$\langle\ln (A)\rangle_{\text {break }}$ & $0.75 \pm 0.05$ & $0.26 \pm 0.10$ & $0.05+0.22 /-\infty$ & $0.08 \pm 0.15$ \\
\hline$\chi^{2} / n d f$ & $7.4 / 9$ & $1.23 / 6$ & $3.37 / 6$ & $4.22 / 8$ \\
\hline
\end{tabular}

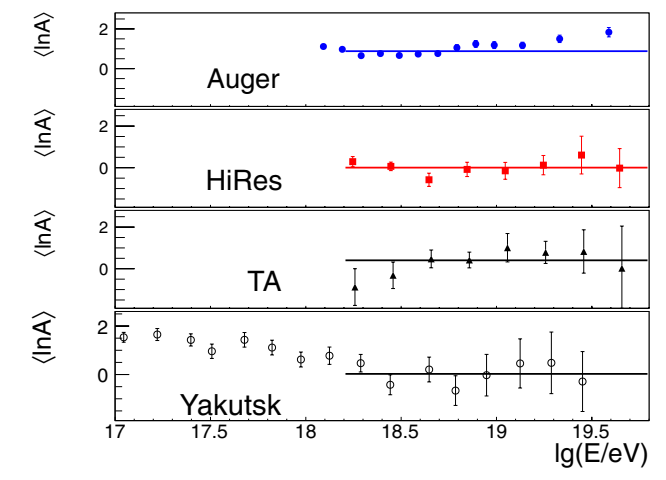

(a) fit to a horizontal line (constant composition).

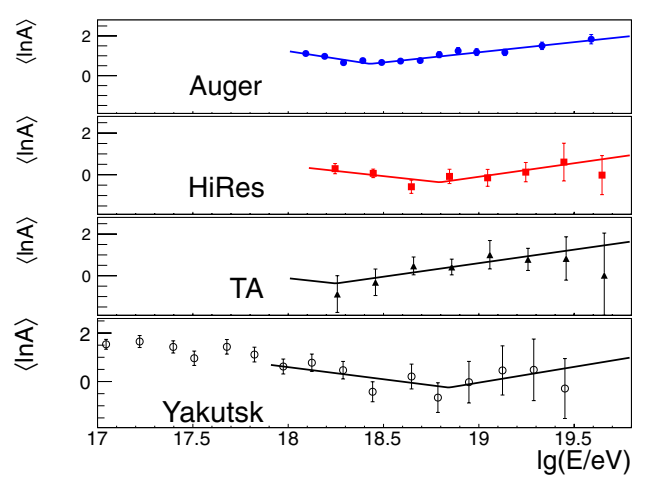

(b) fit to a broken line (changing composition).

Figure 12. Evaluation of the average composition $(\langle\ln A\rangle)$ estimated using SIBYLL as a function of energy. Two composition models are evaluated, a constant composition (as suggested by HiRes and TA) and a changing composition with a break (as suggested by Auger). The results of the fits are summarized in Tables 2 and 3 .

Figure 12(a) shows the test of the HiRes model (a fit to a horizontal line). A horizontal line means constant composition in this plot. The large $\chi^{2} / n d f$ resulting from the fit of the Auger data $\left(\chi^{2} / n d f=137 / 10\right)$ indicates that Auger data does not favor a constant composition model, but all other experiments have $\chi^{2} / n d f$ values embracing the constant composition model (see Table 2).

Figure 12(b) shows the test of the Auger model (a fit to a broken line). The fitted parameters are only the energy and $\langle\ln \mathrm{A}\rangle$ values at which the lines break. The slopes before and after the breaking point are fixed to the results of the Auger fit. The $\chi^{2} / n d f$ values for these fits are small. However, the Auger energy and $\langle\ln \mathrm{A}\rangle$ for the break point is not statistically compatible with the break points fitted by HiRes, TA or Yakutsk (see Table 3). Further, studies (exploring the effect of different interaction models) and more statistics in the Northern Hemisphere are required to establish the level of compatibility between Southern and Northern Hemispheres.

\section{OTHER OBSERVATIONS SENSITIVE TO MASS COMPOSITION}

Apart from $X_{\max }$ observations, other shower observables can also provide information of the average composition. Yakutsk uses an array of muon detectors [5] to measure muon signals at ground level. Auger uses its ground array of water Cherenkov tanks to measure the signal asymmetries around the 


\section{UHECR 2012}

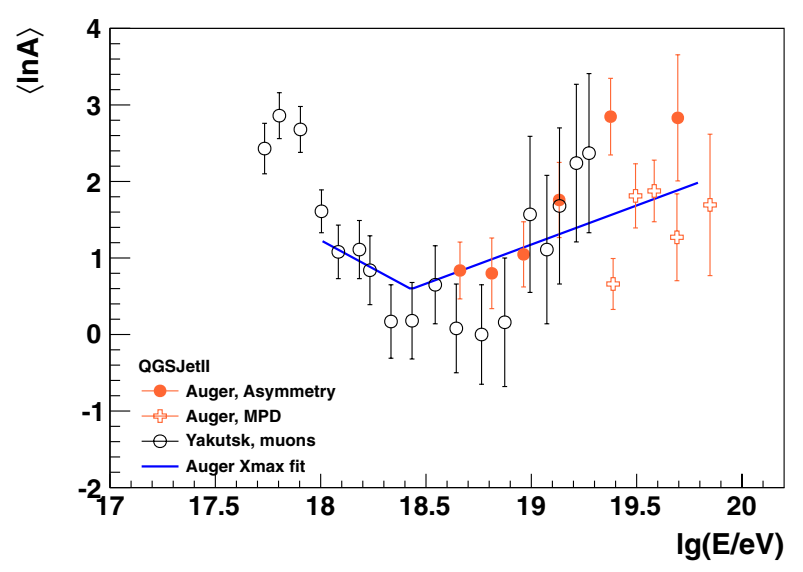

Figure 13. Average composition estimated using other (other than $X_{\max }$ ) shower observables. Open circles are using muon detectors from the Yakutsk experiment [5], solid circles use the observed shower asymmetries around the core with the Auger SD [21], and open crosses are using the estimated muon production depth maximum with the Auger SD.

shower core, and to estimate the muon production depth (MPD) maximum. These observations together with the assistance of Monte Carlo simulations of the detector and hadronic interaction models provide measurements of the average composition $(\langle\ln \mathrm{A}\rangle)$.

Figure 13 shows the average composition as a function of energy estimated using the muon detectors from the Yakutsk experiment, and the Auger ground array. For comparison purposes we have also included the broken lines fitted to the composition estimated using the $X_{\max }$ observations from Auger. For all these estimates of the average composition the model QGSJet-II has been used.

Despite some systematic difference between measurements from Auger $X_{\max }$ and Yakutsk muons, both observations suggest that the composition becomes lighter up to about $10^{18.5} \mathrm{eV}$ and then it becomes heavier again above this energy. Measurements from Auger asymmetries also suggest that the composition becomes heavier above $10^{18.5} \mathrm{eV}$. Measurements from Auger MPD only expand within a narrow energy range, and they do not provide much information regarding the evolution of the composition as a function of energy.

\section{DISCUSSION}

When comparing the $\langle\ln \mathrm{A}\rangle$ values estimated from the $\left\langle X_{\max }\right\rangle$ observations, results from Auger, TA and Yakutsk are compatible within systematic uncertainties. TA and Yakutsk are also compatible with HiRes. However, Auger and HiRes are not fully compatible within systematic uncertainties.

As shown in Section 7, the level of compatibility between Auger and HiRes (the two higheststatistics observatories) depends on the particular interaction model used to interpret the $\left\langle X_{\max }\right\rangle$ observations. Further experimental data on the high-energy hadronic interactions, e.g. from the LHC [25], would help to refine the current composition picture.

We need more statistics in the Northern Hemisphere (about 3 times the current statistics) in order to provide a conclusive statement to whether or not the composition is changing with energy in this Hemisphere. The current data, while completely consistent with a constant light composition, cannot definitively exclude a changing composition as suggested by Auger. More statistics are also necessary to establish whether there is indeed a difference in the $\operatorname{RMS}\left(X_{\max }\right)$ at higher energies between Auger and Yakutsk (Fig. 3). 
In the Northern Hemisphere HiRes has stopped data taking in 2006, however the hybrid TA observatory with a surface area of approximately $800 \mathrm{~km}^{2}$ will be acquiring additional data for the next several years at least.

Figure 13 shows $\langle\ln \mathrm{A}\rangle$ measurements as a function of energy using different techniques. Despite the systematic differences, the measurements suggest a composition getting lighter at energies up to about $10^{18.5} \mathrm{eV}$ and a composition getting heavier above this energy. The systematic differences between different type of measurements are very sensitive to the particular interaction model used for the interpretation. We showed the results for model QGSJet-II (in Fig. 13), because all experiments had results available using this model.

\section{References}

[1] y J. Abraham et al. [Pierre Auger Coll.], Phys. Rev. Lett. 104 (2010) 091101

[2] L. Cazon and R. Ulrich. arXiv:1203.1781

[3] R. Abbasi et al. [HiRes Coll.], Phys. Rev. Lett. 104 (2010) 161101

[4] C. Jui et al. [TA Coll.], Proc. APS DPF Meeting arXiv:1110.0133

[5] L.G. Dedenko et al. J. Phys. G: Nucl. Part. Phys. 39 (2012) 095202

[6] J. Abraham et al., [Pierre Auger Coll.], NIM A 523 (2004) 50

[7] J. Abraham et al., [Pierre Auger Coll.], NIM A 620 (2010) 227

[8] R. U. Abbasi et al., Astropart. Phys. 23 (2005) 157

[9] H. Tokuno et al., NIM A 676 (2012) 54-65, and NIM A 689 (2012) 87

[10] M. Unger [Pierre Auger Coll.], Nucl. Phys. B, Proc. Suppl. 190 (2009) 240

[11] J. Bellido [Pierre Auger Coll.], Proc. XXth Rencontres de Blois (2009), arXiv:0901.3389

[12] D. Heck and J. Knapp, Forschungszentrum Karlsruhe, Tech. Report, (2001)

[13] N. N. Kalmykov and S. S. Ostapchenko, Phys. At. Nucl. 56 (1993) 346

[14] S. Ostapchenko, Nucl. Phys. B, Proc. Suppl. 151, 143 (2006)

[15] R. Fletcher et al., Phys. Rev. D 50 (1994) 5710

[16] R. Engel et al., in Proc. 26th Intl. Cosmic Ray Conference, Salt Lake City, Utah, (1999)

[17] S.P. Knurenko et al., Proc. 27th ICRC, Hamburg 1 (2001), 157

[18] A.M. Hillas, J.R. Patterson. J.Phys.G:Nucl.Phys. 9 (1983), 323

[19] P. Facal for the Pierre Auger Coll. ICRC 2011, arXiv:1107.4804

[20] E.G. Berezhko et al. Astroparticle Physics 36 (2012) 31

[21] D. Garcia-Pinto for the Pierre Auger Coll. ICRC 2011, arXiv:1107.4804

[22] Energy spectrum working group report, this meeting

[23] J. Abraham et al. [Pierre Auger Coll.], Phys. Lett. B 685 (2010) 239

[24] V. de Souza for the Pierre Auger Coll. this meeting

[25] Review of modeling and description of air showers working group report, this meeting 